\title{
A Mixed Finite Element Formulation for the Conservative Fractional Diffusion Equations
}

\author{
Suxiang Yang ${ }^{1,2}$ and Huanzhen Chen ${ }^{1}$ \\ ${ }^{1}$ School of Mathematical Sciences, Shandong Normal University, Jinan 250014, China \\ ${ }^{2}$ School of Mathematics and Quantitative Economics, Shandong University of Finance and Economics, Jinan 250014, China \\ Correspondence should be addressed to Huanzhen Chen; chhzh@sdnu.edu.cn
}

Received 27 April 2016; Accepted 30 June 2016

Academic Editor: Ming Mei

Copyright (c) 2016 S. Yang and H. Chen. This is an open access article distributed under the Creative Commons Attribution License, which permits unrestricted use, distribution, and reproduction in any medium, provided the original work is properly cited.

\begin{abstract}
We consider a boundary-value problem of one-side conservative elliptic equation involving Riemann-Liouville fractional integral. The appearance of the singular term in the solution leads to lower regularity of the solution of the equation, so to the lower order convergence rate for the numerical solution. In this paper, by the dividing of equation, we drop the lower regularity term in the solution successfully and get a new fractional elliptic equation which has full regularity. We present a theoretical framework of mixed finite element approximation to the new fractional elliptic equation and derive the error estimates for unknown function, its derivative, and fractional-order flux. Some numerical results are illustrated to confirm the optimal error estimates.
\end{abstract}

\section{Introduction}

We consider the following one-side conservative diffusion problems of order $2-\beta$ :

$$
\begin{aligned}
-D\left\{K_{0} I_{x}^{\beta} D u\right\}(x) & =f(x), \quad x \in \Omega:=(0,1), \\
u(0) & =0, \\
u(1) & =0,
\end{aligned}
$$

where $D$ is the first-order derivative operator, ${ }_{0} I_{x}^{\beta}$, defined by (2), refers to the left Riemann-Liouville fractional integral of order $\beta \in[0,1), K$ is a positive constant diffusive coefficient, and $f(x) \in L^{2}(\Omega)$ is the source or sink term. Equations (la) and (1b) reduce to the classical second-order diffusion equation when $\beta=0$.

Equations (1a) and (1b), delicately derived from random walk model [1], fractional conservation of mass [2], and the impact of boundary [3], describe subsurface fluid flow and solute transport processes taking place in a multiscale heterogeneous medium/aquifer (see [4] and the references cited therein). These processes exhibit anomalous diffusion or non-Fickian diffusion that cannot be modeled adequately and properly by classical second-order diffusion equation and thus have contracted considerable attention in practical applications.

Since the analytic solutions to general fractional partial differential equations are rarely available, one has to turn to numerical methods in general. In the last decade, a number of numerical methods, for example, finite difference method [5], finite volume method [6], spectral method [7], fast difference method [8], finite element method [9-13], and PetrovGalerkin formulation [14-18], have been developed consecutively for space-fractional partial differential equations.

Considering the conservation property of the diffusion processes and the application of the fractional-order flux $p=-K_{0} I_{x}^{\beta} D u$ in engineering, an ideal numerical procedure should recognize both the unknown function and its flux and obey the conservation of mass to reflect the physical character of the diffusion model ((1a) and (1b)). Hence, one should involve the state variable $u$ and the fractional-order flux $p=-K_{0} I_{x}^{\beta} D u$ to form a saddle-point formulation then design a locally conservative numerical procedure. For this purpose, by introducing two intermediate variables $q=D u$ and the flux $p=-K_{0} I_{x}^{\beta} D u$, Chen and Wang [19] proposed a saddle-point framework and developed a locally conservative expanded mixed finite element method recently. Although a rigorous numerical analysis theory was established in [19], the 
convergence rates for the unknown $u$ and the flux $p$ are not optimal with respect to regularity requirement for sufficiently smooth solution $u$. In particular, for the solution $u$ only in $H^{1-\beta+\gamma}(\Omega)$ with $\gamma \in[\beta / 2,1 / 2)$, the convergence rates are heavily destroyed to $\gamma-\beta / 2$, due to the appearance of the singular term $x^{1-\beta}$.

The aim of this paper is to express the solution of (1a) and (1b) by a full-regular solution satisfying a general fractional diffusion equation with $f$-dependent right-hand side term and a $x^{1-\beta}$-type solution satisfying an analytic-solved fractional equation. Then, the expanded mixed finite element method proposed in [19] can be applied to solve the $f$ dependent fractional equation and obtain good error estimates whatever the regularity of the solution $u$ is.

The rest of the paper is organized as follows. In Section 2, we revisit the fractional differential operators and their properties. In Section 3, we split the fractional diffusion equations (1a) and (1b) into two equations: one is an $f$-dependent fractional diffusion equation that permits a fully regular solution, and the other is an analytic-solved fractional equation from which the $x^{1-\beta}$-type analytic solution is easily solved. By doing so, the solution $u$ of (la) and (1b) is expressed as the sum of the $f$-dependent solution and the $x^{1-\beta}$-type solution. We are devoted to discretizing the $f$-dependent fractional equation by the expanded mixed finite element procedure in Section 4 and deriving the related error estimates in Section 5. Obviously, the error estimates for the original fractional diffusion equations (1a) and (1b) obey those for the $f$-dependent fractional equation since the $x^{1-\beta}$-type solution is analytically solved. In Section 6, numerical experiments are performed to confirm our theoretical findings.

\section{Fractional Operators and Fractional Sobolev Spaces}

In this section, we will recall the definitions and properties of Riemann-Liouville fractional differential operators and fractional Sobolev spaces.

Definition 1 (see [20-22]). Let $\alpha>0$. The left and right Riemann-Liouville fractional integrals of order $\alpha$ are defined by

$$
\begin{aligned}
& { }_{0} I_{x}^{\alpha} u=\frac{1}{\Gamma(\alpha)} \int_{0}^{x}(x-t)^{\alpha-1} u(t) d t \\
& { }_{x} I_{1}^{\alpha} u=\frac{1}{\Gamma(\alpha)} \int_{x}^{1}(t-x)^{\alpha-1} u(t) d t
\end{aligned}
$$

where $\Gamma(\cdot)$ is Gamma function.

Definition 2 (see [20-22]). Let $\alpha>0$ with $n-1<\alpha \leq n$. The left and right Riemann-Liouville fractional derivatives of order $\alpha$ are

$$
\begin{aligned}
& { }_{0} D_{x}^{\alpha} u=\frac{d^{n}}{d x^{n}}\left({ }_{0} I_{x}^{n-\alpha} u\right), \\
& { }_{x} D_{1}^{\alpha} u=(-1)^{n} \frac{d^{n}}{d x^{n}}\left({ }_{x} I_{1}^{n-\alpha} u\right) .
\end{aligned}
$$

We note that fractional integral operator [20-22] satisfies semigroup property; that is, for $\alpha, \beta>0$,

$$
\begin{aligned}
& { }_{0} I_{x}^{\alpha}{ }_{0} I_{x}^{\beta} u={ }_{0} I_{x}^{\alpha+\beta} u, \\
& { }_{x} I_{1}^{\alpha}{ }_{x} I_{1}^{\beta} u={ }_{x} I_{1}^{\alpha+\beta} u,
\end{aligned}
$$

$$
\forall u \in L^{2}(\Omega),
$$

and adjoint property

$$
\left({ }_{0} I_{x}^{\alpha} u, v\right)=\left(u,{ }_{x} I_{1}^{\alpha} v\right), \quad \forall u, v \in L^{2}(\Omega) .
$$

We also observe that the left (right) fractional derivative operator is a left inverse of the left (right) fractional integral operator [20-22]:

$$
\begin{aligned}
& { }_{0} D_{x 0}^{\alpha} I_{x}^{\alpha} u=u, \\
& { }_{x} D_{1 \quad x}^{\alpha} I_{1}^{\alpha} u=u,
\end{aligned}
$$

$$
\forall u \in L^{2}(\Omega) .
$$

We introduce the fractional derivative spaces and present their well-established equivalence to fractional-order Sobolev spaces $[9,19,23]$.

Definition 3 (see [9]). For $\mu>0$, define the norm

$$
\|u\|_{J_{L}^{\mu}(\mathbb{R})}:=\left(\|u\|^{2}+|u|_{J_{L}^{\mu}(\mathbb{R})}^{2}\right)^{1 / 2}
$$

with seminorm

$$
|u|_{J_{L}^{\mu}(\mathbb{R})}:=\left\|_{0} D_{x}^{\mu} u\right\| .
$$

Let $J_{L}^{\mu}(\mathbb{R})$ be the left fractional derivative space defined as the closures of $C_{0}^{\infty}(\mathbb{R})$ with respect to norm $\|\cdot\|_{J_{L}^{\mu}(\mathbb{R})}$.

Definition 4 (see [23]). For $\mu>0$, define the norm

$$
\|u\|_{J_{L}^{-\mu}(\mathbb{R})}:=\left\|I_{x}^{\mu} u\right\|,
$$

and let $J_{L}^{-\mu}(\mathbb{R})$ denote the closures of $C_{0}^{\infty}(\mathbb{R})$ with respect to norm $\|\cdot\|_{J_{L}^{-\mu}(\mathbb{R})}$.

The right fractional derivative spaces $J_{R}^{\mu}(\mathbb{R})$ and $J_{R}^{-\mu}(\mathbb{R})$ and their respective norms are defined analogously.

Definition 5 (see [9]). For $\mu>0$, define the norm

$$
\|u\|_{H^{\mu}(\mathbb{R})}:=\left(\|u\|^{2}+|u|_{H^{\mu}(\mathbb{R})}^{2}\right)^{1 / 2},
$$

with seminorm

$$
|u|_{H^{\mu}(\mathbb{R})}:=\left\||\omega|^{\mu} \widehat{u}(\omega)\right\|,
$$

where $\widehat{u}(\omega)$ denotes the Fourier transform of $u$

$$
\widehat{u}(\omega)=\int_{\mathbb{R}} e^{-i \omega x} u(x) d x .
$$

Let $H^{\mu}(\mathbb{R})$ be the left fractional derivative space defined as the closures of $C_{0}^{\infty}(\mathbb{R})$ with respect to norm $\|\cdot\|_{H^{\mu}(\mathbb{R})}$. 
Definition 6 (see [23]). For $\mu>0$, define the norm

$$
\|u\|_{H^{-\mu}(\mathbb{R})}:=\left\||\omega|^{-\mu} \widehat{u}(\omega)\right\|,
$$

and let $H^{-\mu}(\mathbb{R})$ denote the closures of $C_{0}^{\infty}(\mathbb{R})$ with respect to norm $\|\cdot\|_{H^{-\mu}(\mathbb{R})}$.

We then reiterate the equivalence theories established in $[9,23]$ for the fractional derivative spaces.

Lemma 7 (see [9]). Let $\mu>0$ and $\mu \neq n-1 / 2, n=1,2, \ldots$. Then, $J_{L}^{\mu}(\mathbb{R}), J_{R}^{\mu}(\mathbb{R})$, and the fractional-order Sobolev spaces $H^{\mu}(\mathbb{R})$ are equal, with equivalent seminorms and norms.

Lemma 8 (see [23]). Let $\mu>0$. Then, $J_{L}^{-\mu}(\mathbb{R}), J_{R}^{-\mu}(\mathbb{R})$, and the negative fractional-order Sobolev spaces $H^{-\mu}(\mathbb{R})$, the dual space of $H^{\mu}(\mathbb{R})$, are equal with equivalent norms.

We now restrict the fractional derivative spaces to $\Omega$.

Definition 9 (see [9]). Let $\mu>0$. Define the spaces $J_{L, 0}^{\mu}(\Omega)$, $J_{R, 0}^{\mu}(\Omega)$, and $H_{0}^{\mu}(\Omega)$ as the closures of $C_{0}^{\infty}(\Omega)$ under their respective norms.

Definition 10 (see [23]). Let $\mu>0$. Define the spaces $J_{L}^{-\mu}(\Omega)$, $J_{R}^{-\mu}(\Omega)$, and $H^{-\mu}(\Omega)$ as the closures of $C_{0}^{\infty}(\Omega)$ under their respective norms.

Lemma 11 (see [9]). Let $\mu>0$ and $\mu \neq n-1 / 2, n=1,2, \ldots$. Then, $J_{L, 0}^{\mu}(\Omega), J_{R, 0}^{\mu}(\Omega)$, and the fractional-order Sobolev spaces $H_{0}^{\mu}(\Omega)$ are equal, with equivalent seminorms and norms.

Lemma 12 (see [19]). Let $\mu>0$. Then, $J_{L}^{-\mu}(\Omega), J_{R}^{-\mu}(\Omega)$, and the negative fractional-order Sobolev spaces $H^{-\mu}(\Omega)$, the dual space of $H_{0}^{\mu}(\Omega)$, are equal with equivalent norms.

For simplicity of presentation, we use $\|\cdot\|_{\mu}$ and $|\cdot|_{\mu}$ to stand for the norms and seminorms of fractional-order Sobolev spaces $H^{\mu}(\Omega)$ and $\|\cdot\|_{-\mu}$ to stand for the norms of negative fractional-order Sobolev spaces $H^{-\mu}(\Omega)$, respectively.

\section{Decomposition of (1a) and (1b)}

In this section, we shall split the fractional diffusion equations (1a) and (1b) into an $f$-dependent fractional diffusion equation that permits a fully regular solution and an analyticsolved fractional equation from which the $x^{1-\beta}$-type analytic solution is easily solved.

Theorem 13. Let $u$ be the solution to the fractional diffusion problem ((1a) and (1b)) with right term $f(x) \in L^{2}(\Omega)$. Then,

(1) $u$ can be expressed as

$$
u=u^{r}+u^{s},
$$

where $u^{r}$ and $u^{s}$ are the solutions to the following fractional problems

$$
\begin{aligned}
-D\left\{K_{0} I_{x}^{\beta} D u^{r}\right\}(x) & =f^{r}, \quad x \in \Omega, \\
u^{r}(0) & =0, \\
u^{r}(1) & =0,
\end{aligned}
$$

$$
\begin{aligned}
-D\left\{K_{0} I_{x}^{\beta} D u^{s}\right\}(x) & =\frac{2 K M_{0}}{\Gamma(1+\beta)} x^{\beta}, \quad x \in \Omega, \\
u^{s}(0) & =0, \\
u^{s}(1) & =0 ;
\end{aligned}
$$

here $f^{r}=f(x)-\left(2 K M_{0} / \Gamma(1+\beta)\right) x^{\beta}$ with $M_{0}=$ ${ }_{0} I_{x}{ }_{0} D_{x}^{\beta} I_{x}(f / K)(1)$;

(2) let $p:=-K_{0} I_{x}^{\beta} D u$ and $p^{r}:=-K_{0} I_{x}^{\beta} D u^{r}$ be fractional-order flux. Then, $u \in H_{0}^{1-\beta+\gamma}(\Omega)$ with $\beta / 2 \leq \gamma<$ $1 / 2, p \in H^{1}(\Omega), u^{r} \in H_{0}^{2-\beta}(\Omega), p^{r} \in H^{1}(\Omega)$; further,

$$
\begin{gathered}
\|u\|_{1-\beta+\gamma}+\|p\|_{1} \leq C\|f\|, \\
\left\|u^{r}\right\|_{2-\beta}+\left\|p^{r}\right\|_{1} \leq C\|f\| .
\end{gathered}
$$

Proof. The solution and the regularity of the fractional diffusion equations (1a) and (1b) are given in [19], and the proof is provided only for completeness. Introducing two intermediate variables $q:=D u$ and $p:=-K_{0} I_{x}^{\beta} q,((1 \mathrm{a})$ and $(1 \mathrm{~b}))$ can be rewritten as

$$
\begin{aligned}
D u & =q, \\
0_{x} I_{x}^{\beta} q & =-\frac{p}{K}, \\
D p & =f .
\end{aligned}
$$

Solving (19c) to obtain

$$
p=p(0)+{ }_{0} I_{x} f
$$

and noting ${ }_{0} I_{x} f \in H^{1}(\Omega)$ for $f \in L^{2}(\Omega)$, we conclude that $p \in H^{1}(\Omega)$ and $\|p\|_{1} \leq C\|f\|$.

Solving (19a) and applying ${ }_{0} D_{x}^{\beta}$ to both sides of (19b), we deduce

$$
u=u(0)+{ }_{0} I_{x} q=u(0)-{ }_{0} I_{x}{ }_{0} D_{x}^{\beta}\left(\frac{p}{K}\right) .
$$

Substituting (20) into the above equation and noting the boundary condition $u(0)=0$, we get

$$
u=-\frac{p(0)}{K \Gamma(2-\beta)} x^{1-\beta}-{ }_{0} I_{x}{ }_{0} D_{x}^{\beta}{ }_{0} I_{x}\left(\frac{f}{K}\right) .
$$

Enforcing the boundary condition $u(1)=0$ to the above equation, we obtain $-p(0) / K \Gamma(2-\beta)={ }_{0} I_{x}{ }_{0} D_{x}^{\beta}{ }_{0} I_{x}(f /$ $K)(1):=M_{0}$, which leads to the solution of (1a) and (1b):

$$
u=M_{0} x^{1-\beta}-{ }_{0} I_{x}{ }_{0} D_{x}^{\beta}{ }_{0} I_{x}\left(\frac{f}{K}\right) .
$$

Next, we are to solve (16a) and (16b) and (17a) and (17b). Substituting $f(x)-\left(2 K M_{0} / \Gamma(1+\beta)\right) x^{\beta}$ for $f(x)$ in (22) and noticing the boundary condition $u^{r}(1)=0$, we get the solution of (16a) and (16b):

$$
u^{r}(x)=-{ }_{0} I_{x} 0 D_{x}{ }_{0} I_{x}\left(\frac{f}{K}\right)+M_{0} x^{2} .
$$


Similarly, we get the the solution of (17a) and (17b):

$$
u^{s}(x)=M_{0}\left(x^{1-\beta}-x^{2}\right)
$$

It follows from (23)-(25) that the solution to the fractional problem ((1a) and (1b)) can be split into the solutions of (16a) and (16b) and (17a) and (17b).

Noting that ${ }_{0} D_{x}^{\beta}$ is a bounded operator from fractional Sobolev space $H^{\beta}(\Omega)$ to $L^{2}(\Omega)$ [3], we get the first term ${ }_{-}{ }_{0} I_{x}{ }_{0} D_{x}^{\beta} I_{x}(f / K)$ of solution $u$ in $(23)$ is in $H^{2-\beta}(\Omega)$ with $f \in L^{2}(\Omega)$. The second term $M_{0} x^{1-\beta} \in H^{1-\beta+\gamma}(\Omega)$ with $\beta / 2 \leq \gamma<1 / 2$ [14]. Combining the regularities of two parts and the boundary conditions, we derive $u \in H_{0}^{1-\beta+\gamma}(\Omega)$ and $\|u\|_{1-\beta+\gamma} \leq C\|f\|$. Clearly, the regularity of the second term $M_{0} x^{2} \in H^{2+\gamma}(\Omega)$ is no less than the regularity of the first term in (24). This implies $u^{r} \in H_{0}^{2-\beta}(\Omega), p^{r} \in H^{1}(\Omega)$, and $\left\|u^{r}\right\|_{2-\beta}+\left\|p^{r}\right\|_{1} \leq C\|f\|$.

Remark 14. The decomposition of (1a) and (1b) is not unique in Theorem 13; that is, we can choose any other function $v$, provided that the regularity of $v$ is no less than the regularity of the first term in (24).

Remark 15. From Theorem 13, the regularity of original problem (1a) and (1b) is only $H_{0}^{1-\beta+\gamma}(\Omega)$, due to the presence of the singular term $x^{1-\beta}$, while the regularity to (18) is $H_{0}^{2-\beta}(\Omega)$ with $f(x) \in L^{2}(\Omega)$. In particular, for the right term $f(x) \in$ $H^{l}(\Omega), l \geq 0$, the regularity can be improved to $H_{0}^{l+2-\beta}(\Omega)$ by choosing a proper decomposition. For example, we can replace $x^{2}$ by $x^{m}$ with $m \geq l+2-\beta$ in the decomposition of the solution when $f(x) \in H^{l}(\Omega)$. This is the advantage of the splitting.

Remark 16. Equations (17a) and (17b) is analytic-solvable. Once the regular part $\left(p^{r}, q^{r}, u^{r}\right)$ is determined, the solution $(p, q, u)$ of the source problem ((19a), (19b), and (19c)) (or (1a) and ( $(\mathrm{b}))$ has the representation

$$
\begin{aligned}
& p=p^{r}-K M_{0}\left(\Gamma(2-\beta)-\frac{2}{\Gamma(2+\beta)} x^{1+\beta}\right), \\
& q=q^{r}+M_{0}\left((1-\beta) x^{-\beta}-2 x\right), \\
& u=u^{r}+M_{0}\left(x^{1-\beta}-x^{2}\right) .
\end{aligned}
$$

So we focus on $f$-dependent fractional diffusion equations (16a) and (16b) from now on.

\section{Saddle-Point Formulation and Mixed Finite Element Method}

In this section, we shall formulate a saddle-point variational formulation and establish a mixed finite element formulation to the $f$-dependent fractional diffusion equations (16a) and (16b).
Introduce the fractional-order flux $p^{r}=-K_{0} I_{x}^{\beta} q^{r}$ with $q^{r}=D u^{r}$. Then, we can rewrite (16a) and (16b) into the following mixed form:

$$
\begin{gathered}
D u^{r}=q^{r}, \\
{ }_{0} I_{x}^{\beta} q^{r}=-\frac{p^{r}}{K}, \\
D p^{r}=f^{r} .
\end{gathered}
$$

The mixed variational formulation corresponding to (27a), (27b), and (27c) is to find $\left(p^{r}, q^{r}, u^{r}\right) \in V \times H \times W$ such that

$$
\begin{aligned}
\left(q^{r}, v\right)+\left(u^{r}, D v\right) & =0, \quad v \in V, \\
\left(p^{r}, \sigma\right)+\left(K_{0} I_{x}^{\beta / 2} q^{r},{ }_{x} I_{1}^{\beta / 2} \sigma\right) & =0, \quad \sigma \in H, \\
\left(D p^{r}, w\right) & =\left(f^{r}, w\right), \quad w \in W,
\end{aligned}
$$

where $V=H^{1}(\Omega), H=H^{-\beta / 2}(\Omega)$, and $W=L^{2}(\Omega)$.

Lemma 17 (see [19]). There exists a unique solution $\left(p^{r}, q^{r}, u^{r}\right) \in V \times H \times W$ to the problem (28a), (28b), and (28c).

Next, we shall establish a mixed finite element procedure based on the saddle-point variational formulation (28a), (28b), and (28c).

Let $\Im_{h}$ be a uniform division of $\Omega$ by $I_{i}=\left[x_{i-1}, x_{i}\right], i=$ $1,2, \ldots, M$ with $x_{0}=0, x_{M}=1$, and $h=1 / M$. Let $V_{h} \times H_{h} \times$ $W_{h} \subset V \times H \times W$ denote Raviart-Thomas space [24-27]:

$$
\begin{aligned}
& V_{h}=\left\{v_{h} \in V \cap C^{0}(\bar{\Omega}):\left.v_{h}\right|_{I_{i}} \in P_{k}\left(I_{i}\right), i\right. \\
& =1,2, \ldots, M\}, \quad k \geq 1, \\
& H_{h}=\left\{\sigma_{h} \in H:\left.\sigma_{h}\right|_{I_{i}} \in P_{k}\left(I_{i}\right), \quad i=1,2, \ldots, M\right\}, \\
& \quad k \geq 1, \\
& W_{h}=\left\{w_{h} \in W:\left.w_{h}\right|_{I_{i}} \in P_{k-1}\left(I_{i}\right), \quad i=1,2, \ldots, M\right\}, \\
& \quad k \geq 1,
\end{aligned}
$$

where $P_{k}\left(I_{i}\right)$ is the restriction of the set of all polynomials of a degree not bigger than $k$ on $I_{i}$. Then, the mixed finite element formulation is to find $\left(p_{h}^{r}, q_{h}^{r}, u_{h}^{r}\right) \in V_{h} \times H_{h} \times W_{h}$ such that

$$
\begin{aligned}
\left(q_{h}^{r}, v_{h}\right)+\left(u_{h}^{r}, D v_{h}\right) & =0, \quad v_{h} \in V_{h}, \\
\left(p_{h}^{r}, \sigma_{h}\right)+K\left({ }_{0} I_{x}^{\beta / 2} q_{h}^{r},{ }_{x} I_{1}^{\beta / 2} \sigma_{h}\right) & =0, \quad \sigma_{h} \in H_{h}, \\
\left(D p_{h}^{r}, w_{h}\right) & =\left(f^{r}, w_{h}\right), \\
& w_{h} \in W_{h} .
\end{aligned}
$$


Last, we construct an approximate solution $\left(p_{h}, q_{h}, u_{h}\right)$ to (19a), (19b), and (19c) (or (1a) and (1b)) by

$$
\begin{aligned}
& p_{h}=p_{h}^{r}-K M_{0}\left(\Gamma(2-\beta)-\frac{2}{\Gamma(2+\beta)} x^{1+\beta}\right), \\
& q_{h}=q_{h}^{r}+M_{0}\left((1-\beta) x^{-\beta}-2 x\right), \\
& u_{h}=u_{h}^{r}+M_{0}\left(x^{1-\beta}-x^{2}\right) .
\end{aligned}
$$

\section{Convergence Analysis}

In this section, we are devoted to the convergence analysis. In error analysis below, we shall make use of three projection operators. The first operator is $\Pi_{h}: V \mapsto V_{h}$ defined by

$$
\left(D\left(v-\Pi_{h} v\right), w_{h}\right)=0, \quad \forall w_{h} \in W_{h} .
$$

The other two operators are the $L^{2}$-projections $P_{h}: W \mapsto W_{h}$ and $R_{h}: H \mapsto H_{h}$ :

$$
\begin{aligned}
& \left(u-P_{h} u, w_{h}\right)=0, \quad \forall w_{h} \in W_{h}, \\
& \left(q-R_{h} q, v_{h}\right)=0, \quad \forall v_{h} \in H_{h} .
\end{aligned}
$$

Lemmas 18-20 hold in the integer-order Sobolev spaces (if $n=1$ see [19] and if $n=2,3$ see [24-27]). We can prove that they also hold in the fractional-order spaces by applying standard real interpolation methods [26].

Lemma 18. Let $l$ and $s$ be any real numbers. Assume that $u \in$ $H^{s}(\Omega)$. Then,

$$
\left\|u-P_{h} u\right\|_{-l} \leq C h^{s+l}\|u\|_{s}, \quad 0 \leq l, s \leq k .
$$

Lemma 19. Let $l$ and $s$ be any real numbers. Assume that $q \in$ $H^{s}(\Omega)$. Then,

$$
\left\|q-R_{h} q\right\|_{-l} \leq C h^{s+l}\|q\|_{s}, \quad 0 \leq l, s \leq k+1 .
$$

Lemma 20. Assume that $v \in H^{s}(\Omega)$; then,

$$
\left\|v-\Pi_{h} v\right\|_{\theta} \leq C h^{s-\theta}\|v\|_{s}, \quad 0 \leq \theta \leq 1 .
$$

Now we proceed to the convergence analysis. Let $\xi=$ $\Pi_{h} p^{r}-p_{h}^{r}, \eta=R_{h} q^{r}-q_{h}^{r}$, and $\zeta=P_{h} u^{r}-u_{h}^{r}$; then, from (28a), (28b), and (28c), (30a), (30b), and (30c), (32), and (33), we can get the error equations

$$
\begin{aligned}
\left(\eta, v_{h}\right)+\left(\zeta, D v_{h}\right) & =0, \\
v_{h} & \in V_{h}, \\
\left(p^{r}-p_{h}^{r}, \sigma_{h}\right)+K\left({ }_{0} I_{x}^{\beta / 2}\left(q^{r}-q_{h}^{r}\right),{ }_{x} I_{1}^{\beta / 2} \sigma_{h}\right) & =0, \\
\sigma_{h} & \in H_{h}, \\
\left(D \xi, w_{h}\right) & =0, \\
w_{h} & \in W_{h} .
\end{aligned}
$$

Lemma 21. Suppose that $\xi$ satisfies (37c). Then, the following holds:

$$
\left\|D\left(p^{r}-p_{h}^{r}\right)\right\|=\left\|D\left(p^{r}-\Pi_{h} p^{r}\right)\right\| .
$$

Proof. Set $w_{h}=D \xi \in W_{h}$ in (37c) to derive that $D \xi=0$, which implies $\xi=$ constant. Then, we obtain the desired result.

Lemma 22. There exists a constant $C>0$, such that

$$
\begin{aligned}
\left\|q^{r}-q_{h}^{r}\right\|_{H} \leq & C\left\|q^{r}-R_{h} q^{r}\right\|_{H} \\
& +C h^{1-\beta / 2}\left\|D\left(p^{r}-\Pi_{h} p^{r}\right)\right\| .
\end{aligned}
$$

Proof. Set $v_{h}=\xi$ in (37a). Then, noting $D \xi=0$, we have

$$
(\xi, \eta)=0 .
$$

Set $\sigma_{h}=\eta$ in $(37 \mathrm{~b})$; then,

$$
\begin{aligned}
C_{0}\|\eta\|_{H}^{2} \leq & K \cos \left(\frac{\pi \beta}{2}\right)\left\|{ }_{-\infty} I_{x}^{\beta / 2} \tilde{\eta}\right\| \\
= & K\left({ }_{0} I_{x}^{\beta / 2} \eta,{ }_{x} I_{1}^{\beta / 2} \eta\right) \\
= & -K\left({ }_{0} I_{x}^{\beta / 2}\left(q^{r}-R_{h} q^{r}\right),{ }_{x} I_{1}^{\beta / 2} \eta\right) \\
& -\left(p^{r}-\Pi_{h} p^{r}, \eta\right),
\end{aligned}
$$

where $\left({ }_{0} I_{x}^{\beta / 2} \eta,{ }_{x} I_{1}^{\beta / 2} \eta\right)=\left({ }_{-\infty} I_{x}^{\beta / 2} \widetilde{\eta},{ }_{x} I_{\infty}^{\beta / 2} \widetilde{\eta}\right)=\cos (\pi \beta /$ 2) $\left\|_{-\infty} I_{x}^{\beta / 2} \widetilde{\eta}\right\|, \widetilde{\eta}$ being the extension of $\eta$ by zeros to $\mathbb{R}[23$, Lemma 2.6], is used and $C_{0}=K \cos (\pi \beta / 2)$. Now, we estimate the right-hand side of (41) term by term. For the first term, we get

$$
\begin{gathered}
\left|-K\left({ }_{0} I_{x}^{\beta / 2}\left(q^{r}-R_{h} q^{r}\right),{ }_{x} I_{1}^{\beta / 2} \eta\right)\right| \\
\leq C\left\|q^{r}-R_{h} q^{r}\right\|_{H}\|\eta\|_{H} .
\end{gathered}
$$

For the second term, applying Green's formula, we have

$$
\begin{aligned}
\left(p^{r}-\Pi_{h} p^{r}, \eta\right) & =\left(p^{r}-\Pi_{h} p^{r}, D_{0} I_{x} \eta\right) \\
& =-\left(D\left(p^{r}-\Pi_{h} p^{r}\right),{ }_{0} I_{x} \eta\right),
\end{aligned}
$$

where ${ }_{0} I_{x} \eta(0)={ }_{0} I_{x} \eta(1)=0$ is used. In fact, taking $v_{h}=1$ in $(37 \mathrm{a})$, we get $(\eta, 1)=0$; that is, ${ }_{0} I_{x} \eta(1)=0$. Introduce the $L^{2}$-projection operator $P_{h}^{0}$. Then, the approximation property $\left\|_{0} I_{x} \eta-P_{h}^{0}\left({ }_{0} I_{x} \eta\right)\right\| \leq\left.\left. C h^{1-\beta / 2}\right|_{0} I_{x} \eta\right|_{1-\beta / 2}$ holds. By the property of the $L^{2}$-projection operator $P_{h}^{0}$, (43) can be rewritten as

$$
\begin{aligned}
& \left(p^{r}-\Pi_{h} p^{r}, \eta\right) \\
& \quad=-\left(D\left(p^{r}-\Pi_{h} p^{r}\right),{ }_{0} I_{x} \eta-P_{h}^{0}\left({ }_{0} I_{x} \eta\right)\right) \\
& \quad \leq\left\|D\left(p^{r}-\Pi_{h} p^{r}\right)\right\|\left\|_{0} I_{x} \eta-P_{h}^{0}\left({ }_{0} I_{x} \eta\right)\right\| \\
& \quad \leq\left.\left. C h^{1-\beta / 2}\left\|D\left(p^{r}-\Pi_{h} p^{r}\right)\right\|\right|_{0} I_{x} \eta\right|_{1-\beta / 2} \\
& \quad \leq C h^{1-\beta / 2}\left\|D\left(p^{r}-\Pi_{h} p^{r}\right)\right\|\|\eta\|_{H},
\end{aligned}
$$


where $\left.\left.\right|_{0} I_{x} \eta\right|_{1-\beta / 2} \leq C\left\|_{0} D_{x}^{1-\beta / 2}{ }_{0} I_{x} \eta\right\|=C\left\|_{0} I_{x}^{\beta / 2} \eta\right\| \leq$ $C\|\eta\|_{H}$ is used. Substituting (42) and (44) into (41) yields

$$
\|\eta\|_{H} \leq C h^{1-\beta / 2}\left\|D\left(p^{r}-\Pi_{h} p^{r}\right)\right\|+C\left\|q^{r}-R_{h} q^{r}\right\|_{H} .
$$

In conjunction with triangle inequality, we obtain the desired estimate.

Lemma 23. There exists a constant $C>0$, such that

$$
\left\|p^{r}-p_{h}^{r}\right\| \leq C\left\{\left\|\Pi_{h} p^{r}-p^{r}\right\|+\left\|q^{r}-q_{h}^{r}\right\|_{H}\right\} .
$$

Proof. Taking $\sigma_{h}=\xi$ in error equations (37a), (37b), and (37c), we have

$$
\begin{aligned}
\|\xi\|^{2} & =\left(\Pi_{h} p^{r}-p^{r}, \xi\right)+\left(p^{r}-p_{h}^{r}, \xi\right) \\
& =\left(\Pi_{h} p^{r}-p^{r}, \xi\right)-K\left({ }_{0} I_{x}^{\beta / 2}\left(q^{r}-q_{h}^{r}\right),{ }_{x} I_{1}^{\beta / 2} \xi\right) \\
& \leq\left\|\Pi_{h} p^{r}-p^{r}\right\|\|\xi\|+C\left\|q^{r}-q_{h}^{r}\right\|_{H}\|\xi\|_{H} \\
& \leq\left\|\Pi_{h} p^{r}-p^{r}\right\|\|\xi\|+C\left\|q^{r}-q_{h}^{r}\right\|_{H}\|\xi\| .
\end{aligned}
$$

Then,

$$
\|\xi\| \leq\left\|\Pi_{h} p^{r}-p^{r}\right\|+C\left\|q^{r}-q_{h}^{r}\right\|_{H} .
$$

Together with triangle inequality, we finish the proof of Lemma 23.

Now let us apply the duality argument of Douglas and Roberts to obtain an error estimate in the $L^{2}$-norm.

Lemma 24. There exists a constant $C>0$, such that

$$
\begin{aligned}
\left\|u^{r}-u_{h}^{r}\right\| \leq & \left\|u^{r}-P_{h} u^{r}\right\|+C h^{\gamma-\beta / 2}\left\|q^{r}-q_{h}^{r}\right\|_{H} \\
& +C h^{\gamma-\beta}\left\|p^{r}-p_{h}^{r}\right\| \\
& +C h^{1-\beta+\gamma}\left\|D\left(p^{r}-p_{h}^{r}\right)\right\| .
\end{aligned}
$$

Proof. Let $g \in L^{2}(\Omega)$ and $w \in H_{0}^{1-\beta+\gamma}(\Omega), \beta / 2 \leq \gamma<1 / 2$, such that

$$
\begin{aligned}
-D\left(K_{x} I_{1}^{\beta} D w\right)(x) & =g(x), \quad x \in \Omega=(0,1), \\
w(0) & =0, \\
w(1) & =0 .
\end{aligned}
$$

Then, the regularity estimate $\|w\|_{1-\beta+\gamma} \leq C\|g\|$ holds. Taking $v_{h}=\Pi_{h}\left(K_{x} I_{1}^{\beta} D w\right)$ in (37a), we have

$$
\begin{aligned}
(\zeta, g)= & \left(\zeta,-D\left(K_{x} I_{1}^{\beta} D w\right)\right) \\
= & \left(\zeta,-D \Pi_{h}\left(K_{x} I_{1}^{\beta} D w\right)\right) \\
= & \left(q^{r}-q_{h}^{r}, \Pi_{h}\left(K_{x} I_{1}^{\beta} D w\right)\right) \\
= & \left(q^{r}-q_{h}^{r}, K_{x} I_{1}^{\beta} D w\right) \\
& +\left(q^{r}-q_{h}^{r}, \Pi_{h}\left(K_{x} I_{1}^{\beta} D w\right)-K_{x} I_{1}^{\beta} D w\right) \\
:= & G_{1}+G_{2} .
\end{aligned}
$$

First, we are to estimate the first term $G_{1}$ of (51). Using adjoint property (6), we have

$$
\begin{aligned}
G_{1}= & \left(q^{r}-q_{h}^{r}, K_{x} I_{1}^{\beta} D w\right)=K\left({ }_{0} I_{x}^{\beta}\left(q^{r}-q_{h}^{r}\right), D w\right) \\
= & K\left({ }_{0} I_{x}^{\beta}\left(q^{r}-q_{h}^{r}\right), D w-R_{h}(D w)\right) \\
& +K\left({ }_{0} I_{x}^{\beta}\left(q^{r}-q_{h}^{r}\right), R_{h}(D w)\right) \equiv G_{11}+G_{12} .
\end{aligned}
$$

We use Lemma 19 to estimate $G_{11}$ :

$$
\begin{aligned}
G_{11} & \leq\left\|q^{r}-q_{h}^{r}\right\|_{H}\left\|D w-R_{h}(D w)\right\|_{H} \\
& \leq C h^{\gamma-\beta / 2}\|D w\|_{\gamma-\beta}\left\|q^{r}-q_{h}^{r}\right\|_{H} \\
& \leq C h^{\gamma-\beta / 2}\left\|q^{r}-q_{h}^{r}\right\|_{H}\|w\|_{1-\beta+\gamma} .
\end{aligned}
$$

The estimate of $G_{12}$ is the result of (37c), Lemmas 18 and 19:

$$
\begin{aligned}
G_{12}= & -\left(p^{r}-p_{h}^{r}, R_{h}(D w)\right) \\
= & \left(p^{r}-p_{h}^{r}, D w-R_{h}(D w)\right)+\left(p^{r}-p_{h}^{r}, D w\right) \\
= & \left(p^{r}-p_{h}^{r}, D w-R_{h}(D w)\right)-\left(D\left(p^{r}-p_{h}^{r}\right), w\right) \\
= & \left(p^{r}-p_{h}^{r}, D w-R_{h}(D w)\right) \\
& -\left(D\left(p^{r}-p_{h}^{r}\right), w-P_{h} w\right) \\
& -\left(D\left(p^{r}-p_{h}^{r}\right), P_{h} w\right) \\
\leq & \left\|p^{r}-p_{h}^{r}\right\|\left\|D w-R_{h}(D w)\right\| \\
& +\left\|D\left(p^{r}-p_{h}^{r}\right)\right\|\left\|w-P_{h} w\right\| \\
\leq & C h^{\gamma-\beta}\left\|p^{r}-p_{h}^{r}\right\|\|w\|_{1-\beta+\gamma} \\
& +C h^{1-\beta+\gamma}\left\|D\left(p^{r}-p_{h}^{r}\right)\right\|\|w\|_{1-\beta+\gamma} .
\end{aligned}
$$

Together with above two inequalities, we get the estimate of $G_{1}$ :

$$
\begin{aligned}
G_{1} & \leq C\left\{h^{\gamma-\beta / 2}\left\|q^{r}-q_{h}^{r}\right\|_{H}+h^{\gamma-\beta}\left\|p^{r}-p_{h}^{r}\right\|\right. \\
& \left.+h^{1-\beta+\gamma}\left\|D\left(p^{r}-p_{h}^{r}\right)\right\|\right\}\|w\|_{1-\beta+\gamma} .
\end{aligned}
$$

Thanks to the adjoint property of fractional integral operator, the relationship of $L^{2}$-projection operator $P_{h}$ and $R-T$ operator $\Pi_{h}$, and adjoint problem (50a) and (50b), we get the estimate of $G_{2}$ :

$$
\begin{aligned}
G_{2} & =\left(q^{r}-q_{h}^{r}, \Pi_{h}\left(K_{x} I_{1}^{\beta} D w\right)-K_{x} I_{1}^{\beta} D w\right) \\
& =\left(D_{0} I_{x}\left(q^{r}-q_{h}^{r}\right), \Pi_{h}\left(K_{x} I_{1}^{\beta} D w\right)-K_{x} I_{1}^{\beta} D w\right) \\
& =-\left({ }_{0} I_{x}\left(q^{r}-q_{h}^{r}\right),\right.
\end{aligned}
$$




$$
\begin{aligned}
& \left.D\left(\Pi_{h}\left(K_{x} I_{1}^{\beta} D w\right)-K_{x} I_{1}^{\beta} D w\right)\right) \\
& =-\left({ }_{0} I_{x}\left(q^{r}-q_{h}^{r}\right), P_{h}\left(D\left(K_{x} I_{1}^{\beta} D w\right)\right)\right. \\
& \left.-D\left(K_{x} I_{1}^{\beta} D w\right)\right)=\left({ }_{0} I_{x}\left(q^{r}-q_{h}^{r}\right), P_{h} g-g\right) \\
& =-\left({ }_{0} I_{x}^{\beta / 2}\left(q^{r}-q_{h}^{r}\right),{ }_{x} I_{1}^{1-\beta / 2}\left(P_{h} g-g\right)\right) \leq \| q^{r} \\
& -q_{h}^{r}\left\|_{H}\right\| P_{h} g-g\left\|_{-(1-\beta / 2)} \leq C h^{1-\beta / 2}\right\| g\|\| q^{r}-q_{h}^{r} \|_{H},
\end{aligned}
$$

where ${ }_{0} I_{x}\left(q^{r}-q_{h}^{r}\right)(0)={ }_{0} I_{x}\left(q^{r}-q_{h}^{r}\right)(1)=0$ is used. In fact, taking $v_{h}=1$ in (37a), we derive $\left(q^{r}-q_{h}^{r}, 1\right)=0$; that is, ${ }_{0} I_{x}\left(q^{r}-q_{h}^{r}\right)(1)=0$. Substituting (55) and (56) into (51), and noticing the regularity of the adjoint problem (50a) and (50b), we obtain

$$
\begin{aligned}
\|\zeta\| & \leq C\left\{h^{\gamma-\beta / 2}\left\|q^{r}-q_{h}^{r}\right\|_{H}+h^{\gamma-\beta}\left\|p^{r}-p_{h}^{r}\right\|\right. \\
& \left.+h^{1-\beta+\gamma}\left\|D\left(p^{r}-p_{h}^{r}\right)\right\|\right\} .
\end{aligned}
$$

By triangle inequality, we get the desired result.

By Lemmas 21-24, the properties of Raviart-Thomas operator $\Pi_{h}, L^{2}$ projections $P_{h}$, and $R_{h}$, we obtain the following.

Theorem 25. Let $f(x) \in H^{l}(\Omega)$. Suppose $u^{r}$ is the solution of (27a), (27b), and (27c) and $\left(p_{h}^{r}, q_{h}^{r}, u_{h}^{r}\right) \in V_{h} \times H_{h} \times W_{h}$ satisfies (30a), (30b), and (30c). Then, we can get the following error estimates:

$$
\begin{aligned}
\left\|D\left(p^{r}-p_{h}^{r}\right)\right\| & \leq C h^{\min (l, k)}\|f\|_{l}, \\
\left\|q^{r}-q_{h}^{r}\right\|_{H} & \leq C h^{\min (l+1-\beta / 2, k+1-\beta / 2)}\|f\|_{l}, \\
\left\|p^{r}-p_{h}^{r}\right\| & \leq C h^{\min (l+1-\beta / 2, k+1-\beta / 2)}\|f\|_{l}, \\
\left\|u^{r}-u_{h}^{r}\right\| & \leq C h^{\min (l+1-3 \beta / 2+\gamma, k)}\|f\|_{l} .
\end{aligned}
$$

Proof. Let $f(x) \in H^{l}(\Omega)$. By the regularity of problem (16a) and (16b), we know $u^{r} \in H^{l+2-\beta}(\Omega), q^{r}=D u^{r} \in H^{l+1-\beta}(\Omega)$, and $p^{r} \in H^{l+1}(\Omega)$. Recalling the approximation properties in Lemmas 19 and 20,

$$
\begin{aligned}
\left\|q^{r}-R_{h} q^{r}\right\|_{H} & \leq C h^{\min (l+1-\beta / 2, k+1+\beta / 2)}\left\|q^{r}\right\|_{l+1-\beta}, \\
\left\|D\left(p^{r}-\Pi_{h} p^{r}\right)\right\| & \leq C h^{\min (l, k)}\left\|p^{r}\right\|_{l+1},
\end{aligned}
$$

and combining with Lemmas 21 and 22, we can get the estimates of $\left\|D\left(p^{r}-p_{h}^{r}\right)\right\|$ and $\left\|q^{r}-q_{h}^{r}\right\|_{H}$ :

$$
\begin{aligned}
\| q^{r}- & q_{h}^{r} \|_{H} \\
\leq & C\left\|q^{r}-R_{h} q^{r}\right\|_{H}+C h^{1-\beta / 2}\left\|D\left(p^{r}-\Pi_{h} p^{r}\right)\right\| \\
\leq & C h^{\min (l+1-\beta / 2, k+1+\beta / 2)}\left\|q^{r}\right\|_{l+1-\beta} \\
& +C h^{\min (l+1-\beta / 2, k+1-\beta / 2)}\left\|p^{r}\right\|_{l+1} \\
\leq & C h^{\min (l+1-\beta / 2, k+1-\beta / 2)}\left\{\left\|q^{r}\right\|_{l+1-\beta}+\left\|p^{r}\right\|_{l+1}\right\} \\
\leq & C h^{\min (l+1-\beta / 2, k+1-\beta / 2)}\|f\|_{l} .
\end{aligned}
$$

Analogously, combining the above estimate of $\left\|q^{r}-q_{h}^{r}\right\|_{H}$ and the estimate of $\left\|p^{r}-\Pi_{h} p^{r}\right\|$,

$$
\left\|p^{r}-\Pi_{h} p^{r}\right\| \leq C h^{\min (l+1, k+1)}\left\|p^{r}\right\|_{l+1},
$$

we get from Lemma 23

$$
\begin{aligned}
\| p^{r}- & p_{h}^{r} \| \leq C\left\{\left\|\Pi_{h} p^{r}-p^{r}\right\|+\left\|q^{r}-q_{h}^{r}\right\|_{H}\right\} \\
\leq & C h^{\min (l+1, k+1)}\left\|p^{r}\right\|_{l+1} \\
& +C h^{\min (l+1-\beta / 2, k+1-\beta / 2)}\left\{\left\|q^{r}\right\|_{l+1-\beta}+\left\|p^{r}\right\|_{l+1}\right\} \\
\leq & C h^{\min (l+1-\beta / 2, k+1-\beta / 2)}\left\{\left\|q^{r}\right\|_{l+1-\beta}+\left\|p^{r}\right\|_{l+1}\right\} \\
\leq & C h^{\min (l+1-\beta / 2, k+1-\beta / 2)}\|f\|_{l} .
\end{aligned}
$$

The estimate of $\left\|u^{r}-u_{h}^{r}\right\|$ is a result of Lemmas 21-24 and the following estimate:

$$
\left\|u^{r}-P_{h} u^{r}\right\| \leq C h^{\min (l+2-\beta, k)}\left\|u^{r}\right\|_{l+2-\beta} .
$$

By (26), (31), and Theorem 25, we can derive error estimates for the approximation $\left(p_{h}, q_{h}, u_{h}\right)$.

Theorem 26. Let $f(x) \in H^{l}(\Omega)$. Suppose $(p, q, u)$ is the solution of (19a), (19b), and (19c) (or (1a) and (1b)) and $\left(p_{h}, q_{h}, u_{h}\right)$ satisfies (31). Then, we can get the following error estimates:

$$
\begin{aligned}
\left\|D\left(p-p_{h}\right)\right\| & \leq C h^{\min (l, k)}\|f\|_{l}, \\
\left\|q-q_{h}\right\|_{H} & \leq C h^{\min (l+1-\beta / 2, k+1-\beta / 2)}\|f\|_{l}, \\
\left\|p-p_{h}\right\| & \leq C h^{\min (l+1-\beta / 2, k+1-\beta / 2)}\|f\|_{l}, \\
\left\|u-u_{h}\right\| & \leq C h^{\min (l+1-3 \beta / 2+\gamma, k)}\|f\|_{l} .
\end{aligned}
$$

Remark 27. From Theorem 26 , the estimates $\left\|D\left(p-p_{h}\right)\right\|$ and $\left\|q-q_{h}\right\|_{H}$ are optimal, and the estimates of $\left\|p-p_{h}\right\|$ and $\| u-$ $u_{h} \|$ are suboptimal, even though the regularity of the solution $u$ remains only in $H^{1-\beta+\gamma}(\Omega)$ for $\gamma \in[\beta / 2,1 / 2)$. Hence, in comparison with the standard mixed finite element methods [19], the error rates for $\left\|q-q_{h}\right\|_{H},\left\|p-p_{h}\right\|$, and $\left\|u-u_{h}\right\|$ are only $\gamma-\beta / 2$; in spite of the enough smoothness of the right term $f$, the new approach does yield a higher-order convergence rate.

Remark 28. The estimates of $\left\|p-p_{h}\right\|$ and $\left\|u-u_{h}\right\|$ are only suboptimal, due to the structure of the error equations (37a), (37b), and (37c) and the low global regularity of the adjoint problem (50a) and (50b), used in Nitsches trick. The error equations (37a), (37b), and (37c) involve $u^{r}, p^{r}$, and $q^{r}$ as a whole, so the estimates for $\left\|u^{r}-u_{h}^{r}\right\|$ and $\left\|p^{r}-p_{h}^{r}\right\|$ heavily depend on $\left\|q^{r}-q_{h}^{r}\right\|_{H}$. This may weaken the orders of error estimates. 
TABLE 1: Numerical result of Example 1 on a uniform mesh of size $h$ for $k=1$.

\begin{tabular}{|c|c|c|c|c|c|c|}
\hline$\beta$ & $h$ & $1 / 4$ & $1 / 16$ & $1 / 64$ & $1 / 256$ & Order \\
\hline \multirow{3}{*}{$1 / 4$} & $\left\|u-u_{h}\right\|$ & $2.57 e-2$ & $6.58 e-3$ & $1.65 e-3$ & $4.12 e-4$ & $0.99(1.00)$ \\
\hline & $\left\|p-p_{h}\right\|$ & $1.90 e-2$ & $1.45 e-3$ & $1.14 e-4$ & $9.36 e-6$ & 1.83 (1.63) \\
\hline & $\left\|q-q_{h}\right\|_{H}$ & $1.55 e-2$ & $1.35 e-3$ & $1.24 e-4$ & $1.21 e-5$ & $1.72(1.63)$ \\
\hline \multirow{3}{*}{$1 / 2$} & $\left\|u-u_{h}\right\|$ & $2.64 e-2$ & $6.60 e-3$ & $1.65 e-3$ & $4.12 e-4$ & $1.00(1.00)$ \\
\hline & $\left\|p-p_{h}\right\|$ & $1.72 e-2$ & $1.22 e-3$ & $7.89 e-5$ & $5.01 e-6$ & $1.93(1.75)$ \\
\hline & $\left\|q-q_{h}\right\|_{H}$ & $2.41 e-2$ & $2.13 e-3$ & $1.60 e-4$ & $1.18 e-5$ & 1.77 (1.75) \\
\hline \multirow{3}{*}{$2 / 3$} & $\left\|u-u_{h}\right\|$ & $2.70 e-2$ & $6.61 e-3$ & $1.65 e-3$ & $4.12 e-4$ & $1.01(1.00)$ \\
\hline & $\left\|p-p_{h}\right\|$ & $1.72 e-2$ & $1.22 e-3$ & $7.90 e-5$ & $5.01 e-6$ & $1.96(1.67)$ \\
\hline & $\left\|q-q_{h}\right\|_{H}$ & $2.41 e-2$ & $2.13 e-3$ & $1.61 e-4$ & $1.18 e-5$ & $1.83(1.67)$ \\
\hline
\end{tabular}

TABLE 2: Numerical result of Example 1 on a uniform mesh of size $h$ for $k=2$.

\begin{tabular}{|c|c|c|c|c|c|c|}
\hline$\beta$ & $h$ & $1 / 4$ & $1 / 16$ & $1 / 64$ & $1 / 256$ & Order \\
\hline \multirow{3}{*}{$1 / 4$} & $\left\|u-u_{h}\right\|$ & $7.56 e-3$ & $4.61 e-4$ & $2.88 e-5$ & $1.80 e-6$ & $2.01(1.88)$ \\
\hline & $\left\|p-p_{h}\right\|$ & $2.95 e-2$ & $1.52 e-3$ & $6.92 e-5$ & $3.10 e-6$ & $2.20(1.63)$ \\
\hline & $\left\|q-q_{h}\right\|_{H}$ & $4.44 e-2$ & $2.79 e-3$ & $1.52 e-4$ & $8.12 e-6$ & $2.07(1.63)$ \\
\hline \multirow{3}{*}{$1 / 2$} & $\left\|u-u_{h}\right\|$ & $7.62 e-3$ & $4.61 e-4$ & $2.88 e-5$ & $1.80 e-6$ & $2.00(1.75)$ \\
\hline & $\left\|p-p_{h}\right\|$ & $1.30 e-2$ & $3.89 e-4$ & $1.21 e-5$ & $3.81 e-7$ & $2.51(1.75)$ \\
\hline & $\left\|q-q_{h}\right\|_{H}$ & $3.12 e-2$ & $1.48 e-3$ & $6.70 e-5$ & $3.04 e-6$ & $2.22(1.75)$ \\
\hline \multirow{3}{*}{$2 / 3$} & $\left\|u-u_{h}\right\|$ & $7.94 e-3$ & $4.62 e-4$ & $2.88 e-5$ & $1.80 e-6$ & $2.01(1.67)$ \\
\hline & $\left\|p-p_{h}\right\|$ & $8.73 e-3$ & $1.59 e-4$ & $3.62 e-6$ & $9.66 e-8$ & $2.74(1.83)$ \\
\hline & $\left\|q-q_{h}\right\|_{H}$ & $2.75 e-2$ & $9.84 e-4$ & $3.86 e-5$ & $1.59 e-6$ & $2.34(1.83)$ \\
\hline
\end{tabular}

\section{Numerical Experiments}

In this part, we present some numerical experiments to verify the theoretically proven convergence results. In all the following examples, we let $K=1$. For each example, we consider three different $\beta$ values, that is, $1 / 4,1 / 2$, and $2 / 3$, and present the estimates and the convergence orders of $\left\|u-u_{h}\right\|$, $\left\|p-p_{h}\right\|$, and $\left\|q-q_{h}\right\|_{H^{-\beta / 2}(\Omega)}$, where the number in the bracket under the column order is the theoretical convergence rate derived in Theorem 26.

We consider four examples with the analytic solution of different regularity. The analytic solution of Example 1 is sufficiently smooth, while the others only belong to $H^{1-\beta+\gamma}(\Omega)$ with $\gamma \in[\beta / 2,1 / 2)$, due to the appearance of singular term $x^{1-\beta} \in H^{1-\beta+\gamma}(\Omega)$. So we split (1a) and (1b) into a $f$-dependent fractional diffusion equations (16a) and (16b) and an analytic-solved fractional equation (17a) and (17b) by Theorem 13. Spaces $V_{h} \times H_{h} \times W_{h} \subset V \times H \times W$ choose to be (29) with $k=1$ and $k=2$ for each example.

Example 1. Let $\epsilon \in[0,1 / 2)$; source term $f(x)=-2 x^{\beta} / \Gamma(\beta+$ $1)+6 x^{\beta+1} / \Gamma(\beta+2) \in H^{\beta+\epsilon}(\Omega)$. Then, the analytic solution $u(x)=x^{2}(1-x)$. As $u \in H^{2+\epsilon}(\Omega)$ is very smooth, the result of Theorem 26 predicts almost $O\left(h^{\min (2-\beta / 2, k)}\right)$, $O\left(h^{\min (3 / 2+\beta / 2, k+1-\beta / 2)}\right)$, and $O\left(h^{\min (3 / 2+\beta / 2, k+1-\beta / 2)}\right)$ for $\| u-$ $u_{h}\|\| p-,p_{h} \|$, and $\left\|q-q_{h}\right\|_{H^{-\beta / 2}(\Omega)}$, respectively. Tables 1 and 2 include numerical results for $k=1$ and $k=2$, respectively. From Tables 1 and 2, we can see that they are much higher than the theoretical prediction of Theorem 26.
Example 2. Let source term $f(x)=x^{1+\beta} \in H^{1+\beta+\epsilon}(\Omega)$ for $\epsilon \in[0,1 / 2)$, and $\mathrm{c}_{\beta}=\Gamma(2+\beta) / 6$. Then, the analytic solution $u(x)=c_{\beta}\left(x^{1-\beta}-x^{3}\right)$ only belongs to $H^{1-\beta+\gamma}(\Omega)$ with $\gamma \in$ $[\beta / 2,1 / 2)$, despite the smoothness of the right term $f(x)$. So we split (1a) and (1b) into an $f$-depended fractional diffusion equation with right term $f^{r}(x)=x^{1+\beta}(1-4 x /(2+\beta))$ and analytic solution $u^{r}(x)=c_{\beta}\left(x^{4}-x^{3}\right) \in H^{3+\epsilon}(\Omega)$, and an analytic-solved equation. The numerical results are shown in Tables 3 and 4 . The convergence rates are identical with that for Example 1. This is attributed to the good regularity of the analytic solution $u^{r}$.

Example 3. Let source term $f(x)=1 \in H^{\epsilon}(\Omega)$ for $\epsilon \epsilon$ $[0,1 / 2)$, and constants $c_{\beta}=1 / \Gamma(3-\beta)$. The analytic solution $u(x)=c_{\beta}\left(x^{1-\beta}-x^{2-\beta}\right)$ belongs to $H^{1-\beta+\gamma}(\Omega)$ with $\gamma \in$ $[\beta / 2,1 / 2)$. We split (1a) and (1b) into (16a) and (16b) and (17a) and (17b). We are to solve (16a) and (16b) with right term $f^{r}(x)=1-\left(2 c_{\beta} / \Gamma(1+\beta)\right) x^{\beta}$. The analytic solution $u^{r}(x)=c_{\beta}\left(x^{2}-x^{2-\beta}\right) \in H^{2-\beta+\epsilon}(\Omega)$ is very smooth. The numerical results are shown in Tables 5 and 6 . The same convergence rates are observed, which are much higher than the theoretical prediction of Theorem 26.

Example 4. Let source term $f(x)=x^{-1 / 4}$, which is singular at the origin and belongs to $H^{s}(\Omega)$ for $s \in[0,1 / 4)$. The analytic solution $u(x)=c_{\beta}\left(x^{1-\beta}-x^{7 / 4-\beta}\right)$ only belongs to $H^{1-\beta+\gamma}(\Omega)$ with $\gamma \in[\beta / 2,1 / 2)$ and $c_{\beta}=\Gamma(3 / 4) / \Gamma(11 / 4-\beta)$. We split (1a) and (1b) into two equations (16a) and (16b) and (17a) and 
TABle 3: Numerical result of Example 2 on a uniform mesh of size $h$ for $k=1$.

\begin{tabular}{|c|c|c|c|c|c|c|}
\hline$\beta$ & $h$ & $1 / 4$ & $1 / 16$ & $1 / 64$ & $1 / 256$ & Order \\
\hline \multirow{3}{*}{$1 / 4$} & $\left\|u-u_{h}\right\|$ & $9.60 e-2$ & $2.42 e-2$ & $6.05 e-3$ & $1.51 e-3$ & $0.98(1.00)$ \\
\hline & $\left\|p-p_{h}\right\|$ & $2.06 e-2$ & $1.29 e-3$ & $8.09 e-5$ & $5.06 e-6$ & $2.00(1.88)$ \\
\hline & $\left\|q-q_{h}\right\|_{H}$ & $1.35 e-2$ & $8.15 e-4$ & $4.72 e-5$ & $2.71 e-6$ & $2.05(1.88)$ \\
\hline \multirow{3}{*}{$1 / 2$} & $\left\|u-u_{h}\right\|$ & $9.61 e-2$ & $2.42 e-2$ & $6.05 e-3$ & $1.51 e-3$ & $1.00(1.00)$ \\
\hline & $\left\|p-p_{h}\right\|$ & $1.95 e-2$ & $1.24 e-3$ & $7.78 e-5$ & $4.87 e-6$ & $1.99(1.75)$ \\
\hline & $\left\|q-q_{h}\right\|_{H}$ & $1.28 e-2$ & $9.43 e-4$ & $6.14 e-5$ & $3.88 e-6$ & $1.95(1.75)$ \\
\hline \multirow{3}{*}{$2 / 3$} & $\left\|u-u_{h}\right\|$ & $9.62 e-2$ & $2.42 e-2$ & $6.05 e-3$ & $1.51 e-3$ & $0.99(1.00)$ \\
\hline & $\left\|p-p_{h}\right\|$ & $1.89 e-2$ & $1.23 e-3$ & $7.71 e-5$ & $4.82 e-6$ & 1.99 (1.67) \\
\hline & $\left\|q-q_{h}\right\|_{H}$ & $1.74 e-2$ & $1.36 e-3$ & $8.92 e-5$ & $5.68 e-6$ & $1.93(1.67)$ \\
\hline
\end{tabular}

TABLE 4: Numerical result of Example 2 on a uniform mesh of size $h$ for $k=2$.

\begin{tabular}{|c|c|c|c|c|c|c|}
\hline$\beta$ & $h$ & $1 / 4$ & $1 / 16$ & $1 / 64$ & $1 / 256$ & Order \\
\hline \multirow{3}{*}{$1 / 4$} & $\left\|u-u_{h}\right\|$ & $1.29 e-2$ & $7.98 e-4$ & $4.98 e-5$ & $3.11 e-6$ & $2.00(2.00)$ \\
\hline & $\left\|p-p_{h}\right\|$ & $2.97 e-2$ & $1.52 e-3$ & $6.95 e-5$ & $3.10 e-6$ & $2.20(2.63)$ \\
\hline & $\left\|q-q_{h}\right\|_{H}$ & $4.46 e-2$ & $2.80 e-3$ & $1.52 e-4$ & $8.08 e-6$ & $2.07(2.63)$ \\
\hline \multirow{3}{*}{$1 / 2$} & $\left\|u-u_{h}\right\|$ & $1.29 e-2$ & $7.98 e-4$ & $4.98 e-5$ & $3.11 e-6$ & $2.00(2.00)$ \\
\hline & $\left\|p-p_{h}\right\|$ & $1.29 e-2$ & $3.86 e-4$ & $1.19 e-5$ & $3.71 e-7$ & $2.51(2.75)$ \\
\hline & $\left\|q-q_{h}\right\|_{H}$ & $3.11 e-2$ & $1.48 e-3$ & $6.64 e-5$ & $2.95 e-6$ & $2.23(2.75)$ \\
\hline \multirow{3}{*}{$2 / 3$} & $\left\|u-u_{h}\right\|$ & $1.31 e-2$ & $7.98 e-4$ & $4.98 e-5$ & $3.11 e-6$ & $2.01(2.00)$ \\
\hline & $\left\|p-p_{h}\right\|$ & $8.43 e-3$ & $1.49 e-4$ & $3.24 e-6$ & $7.77 e-8$ & $2.79(2.67)$ \\
\hline & $\left\|q-q_{h}\right\|_{H}$ & $2.70 e-2$ & $9.69 e-4$ & $3.76 e-5$ & $1.47 e-6$ & $2.36(2.67)$ \\
\hline
\end{tabular}

TABLE 5: Numerical result of Example 3 on a uniform mesh of size $h$ for $k=1$.

\begin{tabular}{|c|c|c|c|c|c|c|}
\hline$\beta$ & $h$ & $1 / 4$ & $1 / 16$ & $1 / 64$ & $1 / 256$ & Order \\
\hline \multirow{3}{*}{$1 / 4$} & $\left\|u-u_{h}\right\|$ & $4.95 e-2$ & $1.24 e-2$ & $3.10 e-3$ & $7.76 e-4$ & $1.00(1.00)$ \\
\hline & $\left\|p-p_{h}\right\|$ & $1.88 e-6$ & $1.63 e-7$ & $2.50 e-9$ & $3.85 e-11$ & $2.60(1.38)$ \\
\hline & $\left\|q-q_{h}\right\|_{H}$ & $3.57 e-3$ & $5.33 e-4$ & $7.93 e-5$ & $1.18 e-5$ & $1.37(1.38)$ \\
\hline \multirow{3}{*}{$1 / 2$} & $\left\|u-u_{h}\right\|$ & $5.74 e-2$ & $1.44 e-2$ & $3.60 e-3$ & $9.00 e-4$ & $1.00(1.00)$ \\
\hline & $\left\|p-p_{h}\right\|$ & $1.54 e-4$ & $3.30 e-7$ & $5.60 e-9$ & $8.68 e-11$ & $3.46(1.25)$ \\
\hline & $\left\|q-q_{h}\right\|_{H}$ & $6.70 e-3$ & $1.20 e-3$ & $2.13 e-4$ & $3.76 e-5$ & 1.25 (1.25) \\
\hline \multirow{3}{*}{$2 / 3$} & $\left\|u-u_{h}\right\|$ & $6.25 e-2$ & $1.56 e-2$ & $3.91 e-3$ & $9.78 e-4$ & $1.00(1.00)$ \\
\hline & $\left\|p-p_{h}\right\|$ & $6.56 e-4$ & $1.20 e-6$ & $5.67 e-9$ & $1.06 e-10$ & 3.76 (1.17) \\
\hline & $\left\|q-q_{h}\right\|_{H}$ & $7.67 e-3$ & $1.63 e-3$ & $3.24 e-4$ & $6.44 e-5$ & 1.15 (1.17) \\
\hline
\end{tabular}

TABLE 6: Numerical result of Example 3 on a uniform mesh of size $h$ for $k=2$.

\begin{tabular}{ccccccc}
\hline$\beta$ & $h$ & $1 / 4$ & $1 / 16$ & $1 / 64$ & $1 / 256$ & Order \\
\hline \multirow{3}{*}{$1 / 4$} & $\left\|u-u_{h}\right\|$ & $4.00 e-3$ & $2.58 e-4$ & $1.64 e-5$ & $1.03 e-6$ & $7.88 e-6$ \\
& $\left\|p-p_{h}\right\|$ & $3.88 e-3$ & $5.02 e-4$ & $6.30 e-5$ & $1.99(1.63)$ \\
& $\left\|q-q_{h}\right\|_{H}$ & $6.11 e-3$ & $9.41 e-4$ & $1.40 e-4$ & $2.09 e-5$ & $1.37(1.38)$ \\
\hline \multirow{3}{*}{$1 / 2$} & $\left\|u-u_{h}\right\|$ & $4.14 e-3$ & $3.00 e-4$ & $2.11 e-5$ & $1.45 e-6$ & $6.73 e-6$ \\
& $\left\|p-p_{h}\right\|$ & $3.44 e-2$ & $4.31 e-4$ & $5.38 e-5$ & $4.59 e-5$ & $1.91(1.25)$ \\
& $\left\|q-q_{h}\right\|_{H}$ & $8.33 e-3$ & $1.47 e-3$ & $2.59 e-4$ & $2.14 e-6$ & $1.25(1.25)$ \\
\hline \multirow{3}{*}{$2 / 3$} & $\left\|u-u_{h}\right\|$ & $3.86 e-3$ & $3.24 e-4$ & $2.65 e-5$ & $5.02 e-6$ \\
& $\left\|p-p_{h}\right\|$ & $2.58 e-3$ & $3.20 e-4$ & $4.00 e-5$ & $1.80(1.00)$ \\
& $\left\|q-q_{h}\right\|_{H}$ & $7.94 e-3$ & $1.51 e-3$ & $3.00 e-4$ & $5.94 e-5$ \\
\hline
\end{tabular}


TABLE 7: Numerical result of Example 4 on a uniform mesh of size $h$ for $k=1$.

\begin{tabular}{|c|c|c|c|c|c|c|}
\hline$\beta$ & $h$ & $1 / 4$ & $1 / 16$ & $1 / 64$ & $1 / 256$ & Order \\
\hline \multirow{3}{*}{$1 / 4$} & $\left\|u-u_{h}\right\|$ & $7.65 e-2$ & $1.91 e-2$ & $4.79 e-3$ & $1.20 e-3$ & $1.00(1.00)$ \\
\hline & $\left\|p-p_{h}\right\|$ & $1.61 e-2$ & $3.15 e-3$ & $5.76 e-4$ & $1.0 e 2-4$ & $1.22(1.13)$ \\
\hline & $\left\|q-q_{h}\right\|_{H}$ & $2.53 e-2$ & $5.78 e-3$ & $1.24 e-3$ & $2.63 e-4$ & $1.10(1.13)$ \\
\hline \multirow{3}{*}{$1 / 2$} & $\left\|u-u_{h}\right\|$ & $7.38 e-2$ & $1.84 e-2$ & $4.60 e-3$ & $1.15 e-3$ & $1.00(1.00)$ \\
\hline & $\left\|p-p_{h}\right\|$ & $1.38 e-2$ & $2.70 e-3$ & $4.91 e-4$ & $8.76 e-5$ & $1.22(1.00)$ \\
\hline & $\left\|q-q_{h}\right\|_{H}$ & $2.97 e-2$ & $8.02 e-3$ & $2.04 e-3$ & $5.13 e-4$ & $0.98(1.00)$ \\
\hline \multirow{3}{*}{$2 / 3$} & $\left\|u-u_{h}\right\|$ & $7.27 e-2$ & $1.81 e-2$ & $4.53 e-3$ & $1.13 e-3$ & $1.00(0.75)$ \\
\hline & $\left\|p-p_{h}\right\|$ & $1.26 e-2$ & $2.49 e-3$ & $4.51 e-4$ & $8.03 e-5$ & $1.22(0.92)$ \\
\hline & $\left\|q-q_{h}\right\|_{H}$ & $3.02 e-2$ & $9.51 e-3$ & $2.71 e-3$ & $7.63 e-4$ & $0.88(0.92)$ \\
\hline
\end{tabular}

TABLE 8: Numerical result of Example 4 on a uniform mesh of size $h$ for $k=2$.

\begin{tabular}{|c|c|c|c|c|c|c|}
\hline$\beta$ & $h$ & $1 / 4$ & $1 / 16$ & $1 / 64$ & $1 / 256$ & Order \\
\hline \multirow{3}{*}{$1 / 4$} & $\left\|u-u_{h}\right\|$ & $5.50 e-3$ & $3.99 e-4$ & $2.80 e-5$ & $1.92 e-6$ & $1.91(1.38)$ \\
\hline & $\left\|p-p_{h}\right\|$ & $1.14 e-2$ & $2.05 e-3$ & $3.62 e-4$ & $6.40 e-5$ & $1.25(1.13)$ \\
\hline & $\left\|q-q_{h}\right\|_{H}$ & $1.81 e-2$ & $3.86 e-3$ & $8.12 e-4$ & $1.71 e-4$ & 1.12 (1.13) \\
\hline \multirow{3}{*}{$1 / 2$} & $\left\|u-u_{h}\right\|$ & $3.95 e-3$ & $3.63 e-4$ & $3.27 e-5$ & $2.92 e-6$ & $1.73(1.00)$ \\
\hline & $\left\|p-p_{h}\right\|$ & $3.91 e-2$ & $6.88 e-4$ & $1.22 e-4$ & $2.15 e-5$ & $1.25(1.00)$ \\
\hline & $\left\|q-q_{h}\right\|_{H}$ & $1.14 e-2$ & $2.84 e-3$ & $7.09 e-4$ & $1.77 e-4$ & $1.00(1.00)$ \\
\hline \multirow{3}{*}{$2 / 3$} & $\left\|u-u_{h}\right\|$ & $2.36 e-3$ & $2.65 e-4$ & $2.96 e-5$ & $3.31 e-6$ & $1.58(0.75)$ \\
\hline & $\left\|p-p_{h}\right\|$ & $2.48 e-3$ & $4.33 e-4$ & $7.65 e-5$ & $1.38 e-5$ & $1.25(0.92)$ \\
\hline & $\left\|q-q_{h}\right\|_{H}$ & $7.12 e-3$ & $1.99 e-3$ & $5.60 e-4$ & $1.58 e-4$ & $0.92(0.92)$ \\
\hline
\end{tabular}

(17b). We are to solve (16a) and (16b) with right term $f^{r}(x)=$ $x^{-1 / 4}-\left(2 c_{\beta} / \Gamma(1+\beta)\right) x^{\beta}$ and analytic solution $u^{r}(x)=c_{\beta}\left(x^{2}-\right.$ $\left.x^{7 / 4-\beta}\right) \in H^{7 / 4-\beta+\epsilon}(\Omega)$. The numerical results are shown in Tables 7 and 8 .

Remark 5. From the above four examples, we can see that the error rates for $\left\|q-q_{h}\right\|_{H}$ are identical to the predicted rates of convergence in Theorem 26. However, numerical convergence rates for $\left\|p-p_{h}\right\|$ and $\left\|u-u_{h}\right\|$ are much higher than the theoretical prediction of Theorem 26.

\section{Competing Interests}

The authors declare that they have no competing interests.

\section{Acknowledgments}

This work is supported in part by the National Natural Science Foundation of China under Grant nos. 10971254, 11301311, 11471196, 2014ZRB01849, and 61503214.

\section{References}

[1] D. A. Benson, S. W. Wheatcraft, and M. M. Meerschaert, "The fractional-order governing equation of Levy motion," Water Resources Research, vol. 36, no. 6, pp. 1413-1423, 2000.

[2] S. W. Wheatcraft and M. M. Meerschaert, "Fractional conservation of mass," Advances in Water Resources, vol. 31, no. 10, pp. 1377-1381, 2008.

[3] X. Zhang, M. Lv, J. W. Crawford, and I. M. Young, “The impact of boundary on the fractional advection-dispersion equation for solute transport in soil: defining the fractional dispersive flux with the caputo derivatives," Advances in Water Resources, vol. 30, no. 5, pp. 1205-1217, 2007.

[4] S. P. Neuman and D. M. Tartakovsky, "Perspective on theories of non-Fickian transport in heterogeneous media," Advances in Water Resources, vol. 32, no. 5, pp. 670-680, 2009.

[5] M. M. Meerschaert and C. Tadjeran, "Finite difference approximations for two-sided space-fractional partial differential equations," Applied Numerical Mathematics, vol. 56, no. 1, pp. 80-90, 2006.

[6] Q. Yang, I. Turner, T. Moroney, and F. Liu, "A finite volume scheme with preconditioned Lanczos method for two-dimensional space-fractional reaction-diffusion equations," Applied Mathematical Modelling, vol. 38, no. 15-16, pp. 3755-3762, 2014.

[7] N. Nie, J. Huang, W. Wang, and Y. Tang, "Solving spatialfractional partial differential diffusion equations by spectral method," Journal of Statistical Computation and Simulation, vol. 84, no. 6, pp. 1173-1189, 2014.

[8] H. Wang and T. S. Basu, "A fast finite difference method for two-dimensional space-fractional diffusion equations," SIAM Journal on Scientific Computing, vol. 34, no. 5, pp. A2444A2458, 2012.

[9] V. J. Ervin and J. P. Roop, "Variational formulation for the stationary fractional advection dispersion equation," Numerical Methods for Partial Differential Equations, vol. 22, no. 3, pp. 558576, 2006.

[10] V. J. Ervin and J. P. Roop, "Variational solution of fractional advection dispersion equations on bounded domains in $\mathbb{R}^{d}$," Numerical Methods for Partial Differential Equations, vol. 23, no. 2, pp. 256-281, 2007.

[11] V. J. Ervin, N. Heuer, and J. P. Roop, "Numerical approximation of a time dependent, nonlinear, space-fractional diffusion 
equation," SIAM Journal on Numerical Analysis, vol. 45, no. 2, pp. 572-591, 2007.

[12] H. Wang, D. P. Yang, and S. F. Zhu, "Accuracy of finite element methods for boundary-value problems of steady-state fractional diffusion equations," Journal of Scientific Computing, 2016.

[13] B. T. Jin and Z. Zhou, "A finite element method with singularity reconstruction for fractional boundary value problems," ESAIM. Mathematical Modelling and Numerical Analysis, vol. 49, no. 5, pp. 1261-1283, 2015.

[14] B. Jin, R. Lazarov, J. Pasciak, and W. Rundell, "Variational formulation of problems involving fractional order differential operators," Mathematics of Computation, vol. 84, no. 296, pp. 2665-2700, 2015.

[15] H. Wang and D. P. Yang, "Wellposedness of variable-coefficient conservative fractional elliptic differential equations," SIAM Journal on Numerical Analysis, vol. 51, no. 2, pp. 1088-1107, 2013.

[16] H. Wang, D. Yang, and S. Zhu, "A Petrov-Galerkin finite element method for variable-coefficient fractional diffusion equations," Computer Methods in Applied Mechanics and Engineering, vol. 290, pp. 45-56, 2015.

[17] D. P. Yang and H. Wang, "Wellposedness and regularity of steady-state two-sided variablecoefficient conservative space-fractional diffusion equations," https://arxiv.org/abs/1606 .04912 .

[18] B. Jin, R. Lazarov, and Z. Zhou, "A Petrov-Galerkin finite element method for fractional convection-diffusion equations," SIAM Journal on Numerical Analysis, vol. 54, no. 1, pp. 481-503, 2016.

[19] H. Chen and H. Wang, "Numerical simulation for conservative fractional diffusion equations by an expanded mixed formulation," Journal of Computational and Applied Mathematics, vol. 296, pp. 480-498, 2016.

[20] S. G. Samko, A. A. Kilbas, and O. I. Marichev, Fractional Integrals and Derivatives: Theory and Applications, Gordon and Breach Science, London, UK, 1993.

[21] K. B. Oldham and J. Spanier, The Fractional Calculus, Academic Press, New York, NY, USA, 1974.

[22] I. Podlubny, Fractional Differential Equations, Academic Press, New York, NY, USA, 1999.

[23] W. H. Deng and J. S. Hesthaven, "Local discontinuous Galerkin methods for fractional diffusion equations," ESAIM: Mathematical Modelling and Numerical Analysis, vol. 47, no. 6, pp. 18451864, 2013.

[24] F. Brezzi and M. Fortin, Mixed and Hybrid Finite Element Methods, vol. 15, Springer, New York, NY, USA, 1991.

[25] F. Brezzi, J. Douglas, R. Durán, and M. Fortin, "Mixed finite elements for second order elliptic problems in three variables," Numerische Mathematik, vol. 51, no. 2, pp. 237-250, 1987.

[26] P. A. Raviat and J. M. Thomas, "A mixed finite element method for 2-nd order elliptic problems," in Mathematical Aspects of Finite Element Methods, I. Galligani and E. Magenes, Eds., vol. 606 of Lecture Notes in Mathematics, pp. 292-315, Springer, Berlin, Germany, 1977.

[27] F. Brezzi, J. Douglas Jr., and L. D. Marini, "Two families of mixed finite elements for second order elliptic problems," Numerische Mathematik, vol. 47, no. 2, pp. 217-235, 1985. 


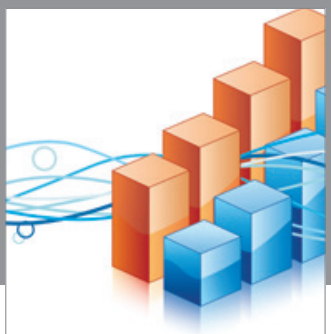

Advances in

Operations Research

vatem alat4

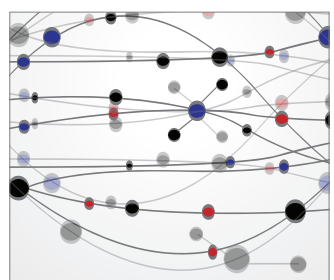

\section{The Scientific} World Journal
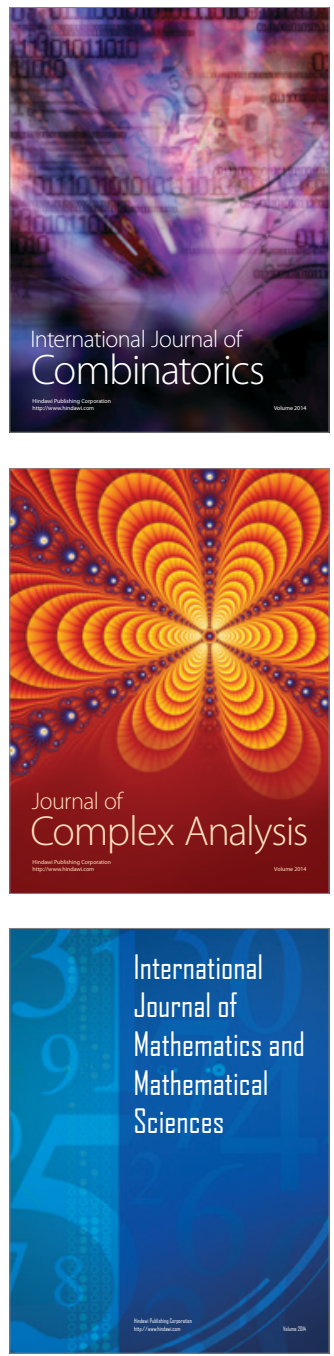
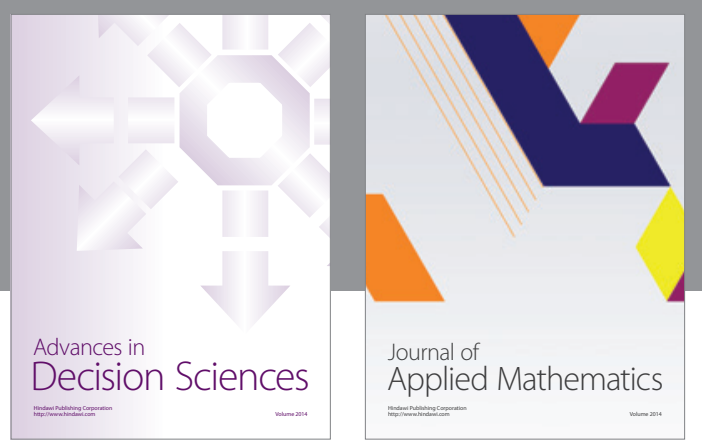

Algebra

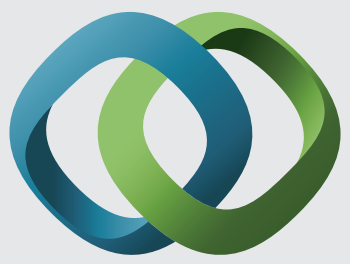

\section{Hindawi}

Submit your manuscripts at

http://www.hindawi.com
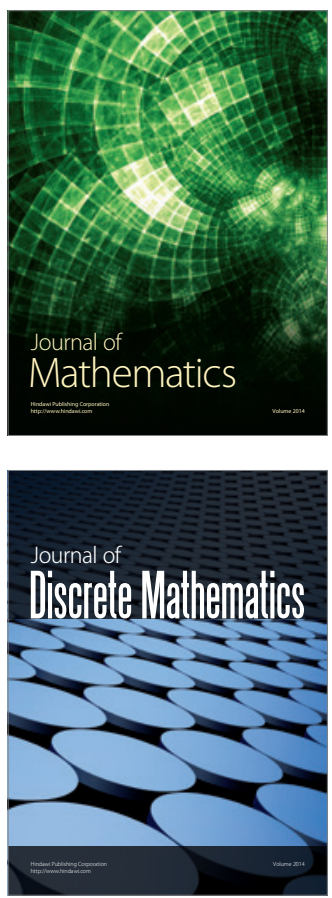

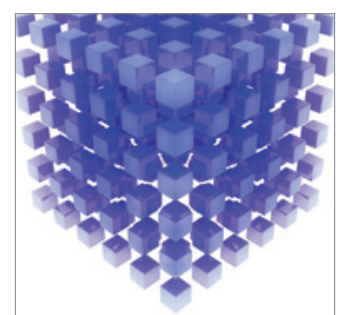

Mathematical Problems in Engineering
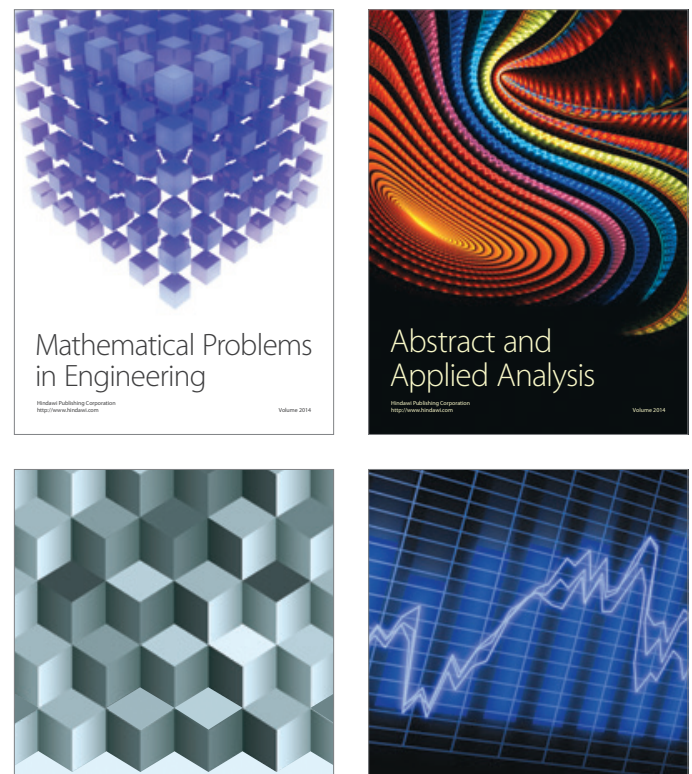

Journal of

Function Spaces

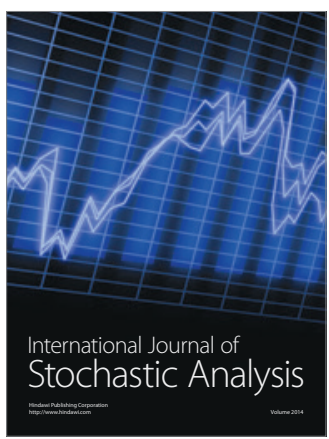

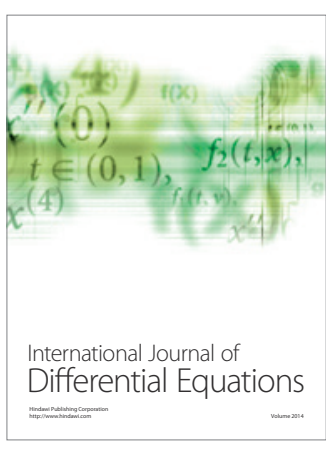
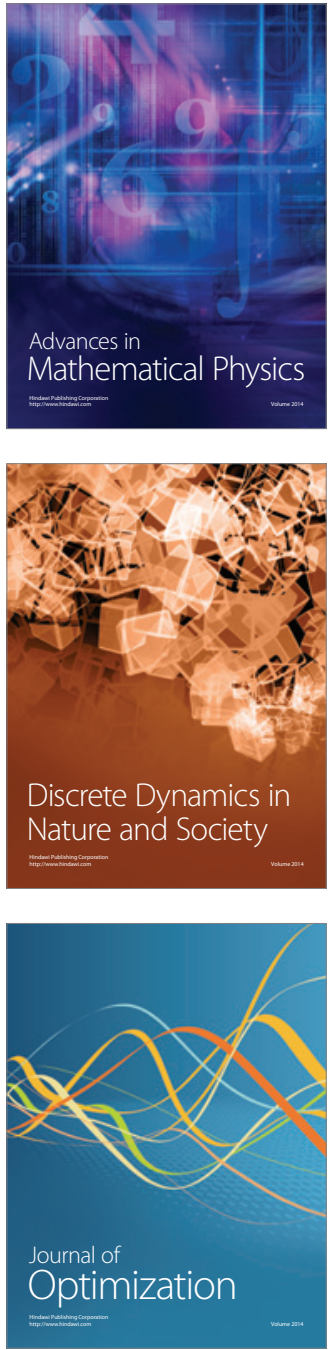